\title{
Estilos de liderazgo directivo y la gestión de conflictos en las instituciones educativas públicas
}

Leadership leadership styles and conflict management in public educational institutions

Lucía Angélica Zegarra Ordoñez

\section{RESUMEN}

Objetivo: Determinar la relación entre los estilos de liderazgo del director y la gestión de conflictos en las instituciones educativas públicas de la Red N 08- UGEL № 04 - Puente Piedra - Lima, 2017. Métodos: Se propuso el enfoque cuantitativo de diseño correlacional, asimismo, se recolectó información mediante un cuestionario que fue aplicado a la muestra conformada por 100 docentes, para conocer sus puntos de vista con respecto a las variables de estudio, de otro lado se garantizó su confiabilidad mediante la prueba de Alfa de Cronbach. Además de aplicar el paquete computacional SPSS versión 21, con el empleo de la fórmula de Rho de Spearman con un margen de error al 5\%. Resultados: La hipótesis general representado por las variables encontró una correlación buena de Rho $=0,626$, ya que la mayoría consideró que es a veces idóneo los estilos de liderazgo directivo, incidiendo así en la gestión de conflictos dentro de la institución educativa considerada a veces adecuada. Con respecto a la primera, segunda y tercera hipótesis específicas, estas alcanzaron una correlación moderada de Rho $=0,505$, Rho $=0,514$ y Rho $=$ 0,474 con respecto a los estilos de liderazgo y relación con las dimensiones manejo de la negociación, comunicación cordial y uso de la mediación respectivamente. Conclusión: Los resultados obtenidos evidencian que la falta de identificación de estilos de liderazgo directivo para cada tipo de situación conflictiva, seguirá generando descontento entre los miembros de la institución en estudio.

Palabras clave: Liderazgo directivo, manejo de la negociación, comunicación cordial, uso de la mediación.

\section{ABSTRACT}

Objective: Determine the relationship between the leadership styles of the director and the management of conflicts in the public educational institutions of the Network $N^{\circ} 08-$ UGEL $N^{\circ} 04$ Puente Piedra - Lima, 2017. Methods: The quantitative design approach was proposed at the same time, information was collected through a questionnaire that was applied to the sample consisting of 100 teachers, to know their points of view regarding the study variables, on the other hand their reliability was guaranteed through the Cronbach's Alpha test. In addition to applying the SPSS software package version 21, with the use of Spearman's Rho formula with a margin of error of $5 \%$. Results: The general hypothesis represented by the variables found a good correlation of Rho $=.626$, since Most of them consider that leadership styles are sometimes appropriate, thus influencing the management of conflicts within the educational institution considered sometimes adequate. With respect to the first, second and third specific hypotheses, these reached a moderate correlation of Rho $=.505$, Rho $=.514$ and $\mathrm{Rho}=.474$ with respect to leadership styles and relationship with the dimensions negotiation management, communication cordial and use of mediation respectively. Conclusion: The results obtained show that the lack of identification of leadership styles for each type of conflictive situation will continue to generate dissatisfaction among the members of the institution under study.

Keywords: Directive leadership, negotiation management, cordial communication, use of mediation.

\footnotetext{
1 Universidad Nacional Mayor de San Marcos. Lima, Perú.
} 


\section{INTRODUCCIÓN}

La existencia de conflictos en las instituciones educativas de nuestro país constituye un fenómeno habitual propio de todo Sistema Educativo Formal. Cada día se escucha o se lee a través de los medios de comunicación los conflictos externos e internos que afectan la imagen del sistema educativo público; muy a menudo los directores y docentes son denunciados por padres de familia o alumnos y viceversa, acusándose mutuamente, creando un impacto negativo en el proceso educativo. Estas situaciones no tienen por qué llegar a ser negativas, pues los conflictos, si se abordan adecuadamente y se les da una solución satisfactoria, adquieren un gran potencial educativo y sirve para fortalecer las relaciones entre personas.

En esta gran tarea, la labor del Director, juega un rol muy importante, pues como líder y orientador tiene que comprometer la máxima participación dentro de un clima de gobernabilidad y concertación para propiciar el clima favorable y el logro de los objetivos educacionales e institucionales, asumir la existencia del conflicto para buscar las alternativas y darle un manejo de forma constructiva. Como lo afirma Alvarado (2003) "El liderazgo es la función de conducir, guiar, dirigir a los colaboradores en base a la fuerza de ideas, del carácter, del talento, la voluntad y la habilidad administrativa hacia el logro de los objetivos institucionales preestablecidos".

En una investigación nacional, realizado por Mena (2015) también se alertó inconvenientes para la resolución de conflictos por parte del director, señalando que estos por lo general no poseen el suficiente dominio de los conocimientos básicos requeridos para una gestión eficiente de conflictos en el Marco de Buen Desempeño del Directivo propuesto por el Ministerio de Educación, asimismo detalló que no cuentan con las habilidades necesarias ni manejan estrategias adecuadas para promover la resolución pacífica de conflictos mediante el diálogo, el consenso y la negociación, teniendo en cuenta la naturaleza específica de los conflictos y las circunstancias concretas en que éstos se producen, de modo que puedan transformarse en oportunidades de aprendizaje.
Las instituciones educativas de nuestro sector atraviesan una serie de problemas al interior de su organización, algunos de ellos son: interferencias externas (Normas incompatibles, injerencia en la autonomía de la gestión), falta de confianza a la autoridad, desconocimiento e incumplimiento de funciones, falta de liderazgo del director, incumplimiento del reglamento interno, falta de transparencia en la gestión, toma de decisiones sin criterio que conllevan a las manifestaciones de conflictos internos, deteriorando el clima institucional, por ende afecta el servicio educativo en la gestión pública.

Se observa que los Directores ejercen diversos estilos de liderazgo, lo cual en alguna medida afecta el manejo adecuado de los conflictos en las instituciones educativas y ésta no sea constructiva. Algunos directores reprimen el conflicto, o evaden y pocos intentan una solución constructiva.

Al respecto, Minedu (2009) indica que la autoridad máxima de un centro educativo es el director y tiene la ardua responsabilidad de saber conducir y proporcionar informe sobre los trámites que realiza, es la persona indicada a la resolución de conflictos que surge dentro del centro educativo; su liderazgo se fortalece cuando practica un liderazgo basado en valores éticos, morales y democráticos.

En este contexto, la hipótesis de estudio fue: Los estilos de liderazgo se asocian significativamente con liderazgo del director y la gestión de conflictos en las instituciones educativas públicas de la Red $N^{\circ} 08-$ UGEL N $^{\circ}$ 04 - Puente Piedra - Lima, 2017. El objetivo fue determinar la relación entre los estilos de liderazgo del director y la gestión de conflictos en las instituciones educativas públicas de la Red N ${ }^{\circ}$ 08- UGEL N04 - Puente Piedra Lima, 2017.

\section{MATERIAL Y MÉTODOS}

Corresponde a la investigación del enfoque cuantitativo, nivel explicativo, ex pos facto, ya que intenta analizar y evaluar la relación entre las variables existentes, es tipo no experimental con un diseño correlacional. La población estuvo conformada por 200 
docentes de la Red $N^{\circ}$ 08-UGEL N ${ }^{\circ} 04$ de Puente Piedra. La muestra estuvo conformada por 100 docentes. Para el tratamiento estadístico, se empleó el paquete computacional SPSS versión 21. Los instrumentos empleados fueron dos cuestionarios para conocer las opiniones de los docentes, uno para cada variable (Estilos de liderazgo directivo y gestión de conflictos), asimismo, se empleará la técnica del coeficiente de correlación de Spearman y así evidenciar de manera estadística el grado de relación y consistencia entre las variables planteadas. El cuestionario para la variable "Estilos de liderazgo directivo" estuvó constituido por 27 ítems en base a una escala de Likert, la cual tuvo por finalidad recopilar su valoración con respecto al a los estilos de liderazgo, a través de la apreciación de cinco dimensiones. El cuestionario para la variable "Gestión de conflictos" estuvo constituido por 21 ítems en base a una escala de Likert, la cual tiene por finalidad recopilar su valoración con respecto a la gestión de conflictos, a través de la apreciación de tres dimensiones.

\section{RESULTADOS}

Se estableció la contrastación de las siguientes hipótesis:

\section{Prueba de hipótesis general}

HGA. Los estilos de liderazgo se asocian significativamente con liderazgo del director y la gestión de conflictos en las instituciones educativas públicas de la Red N 08- UGEL N04 - Puente Piedra - Lima, 2017.

HGO. Los estilos de liderazgo no se asocian significativamente con liderazgo del director y la gestión de conflictos en las instituciones educativas públicas de la Red N 08- UGEL N04 - Puente Piedra - Lima, 2017.

Tabla 1. Correlación de la hipótesis general

\begin{tabular}{lccc} 
& \multicolumn{2}{c}{ Correlaciones } & Gestión de conflictos \\
\hline \multirow{2}{*}{ Rho de } & \multirow{2}{*}{ Estilos de } & Coeficiente de correlación & 0,626 \\
Spearman & liderazgo & Sig. (bilateral) & 0,000 \\
& & $\mathrm{~N}$ & 100 \\
\hline
\end{tabular}

Fuente: Zegarra (2017)

Al aplicar la fórmula de Rho de Spearman, margen de error al $5 \%$, se halló un coeficiente de correlación buena (Rho=0,626; $p<0,01)$. Por lo cual se rechaza la hipótesis nula y se acepta la hipótesis alterna, cuyos resultados fueron determinados por la respuesta de la muestra, en donde la mayoría considera que los estilos de liderazgo inciden en la gestión de conflictos identificado como a veces adecuado.

\section{Contrastación de la primera hipótesis específica}

$\mathrm{H}_{1}$. Los estilos de liderazgo del director se relacionan significativamente con el manejo de la negociación en las instituciones educativas públicas de la Red N $08-U G E L N^{\circ} 04$ - Puente Piedra Lima, 2017.

$\mathrm{H}_{\mathrm{o}}$. Los estilos de liderazgo del director no se relacionan significativamente con el manejo de la negociación en las instituciones educativas públicas de la Red N 08-UGEL N 04 - Puente Piedra Lima, 2017.

Tabla 2. Correlación de la primera hipótesis específica

\begin{tabular}{llcc}
\hline & \multicolumn{2}{c}{ Correlaciones } & Manejo de la Negociación \\
\hline \multirow{2}{*}{ Rho de } & Estilos de & Coeficiente de correlación & 0,505 \\
Spearman & liderazgo & Sig. (bilateral) & 0,000 \\
& & $N$ & 100 \\
\hline
\end{tabular}

Fuente: Zegarra (2017) 
Al aplicar la fórmula de Rho de Spearman, margen de error al $5 \%$, se halló un coeficiente de correlación moderada (Rho $=0,626$; $p<0,01)$. Por lo cual se rechaza la hipótesis nula y se acepta la hipótesis alterna, cuyos resultados fueron determinados por la respuesta de la muestra, en donde la mayoría considera que los estilos de liderazgo inciden en el manejo de la negociación identificado como a veces adecuado.

\section{Contrastación de la segunda hipótesis específica}

$\mathrm{H}_{2}$. Los estilos de liderazgo del director se relacionan significativamente con la comunicación cordial en las instituciones educativas públicas de la Red N 08- UGEL Nº 04 - Puente Piedra - Lima, 2017.

$\mathrm{H}_{\mathrm{o}}$. Los estilos de liderazgo del director no se relacionan significativamente con la comunicación cordial en las instituciones educativas públicas de la Red N 08- UGEL N04 - Puente Piedra - Lima, 2017.

Tabla 3. Correlación de la segunda hipótesis específica

\begin{tabular}{cccc}
\multicolumn{2}{c}{ Correlaciones } & $\begin{array}{c}\text { Comunicación } \\
\text { Cordial }\end{array}$ \\
\hline \multirow{2}{*}{ Rho de } & Estilos de & Coeficiente de correlación & 0,514 \\
Spearman & liderazgo & Sig. (bilateral) & 0,000 \\
& & $\mathrm{~N}$ & 100 \\
\hline
\end{tabular}

Al aplicar la fórmula de Rho de Spearman, margen de error al $5 \%$, se halló un coeficiente de correlación moderada (Rho $=0,514$; $p<0,01)$. Por lo cual se rechaza la hipótesis nula y se acepta la hipótesis alterna, cuyos resultados fueron determinados por la respuesta de la muestra, en donde la mayoría considera que los estilos de liderazgo directivo inciden en la comunicación cordial como a veces adecuado.

\section{Contrastación de la tercera hipótesis específica}

$\mathrm{H}_{3}$. Los estilos de liderazgo del director se relacionan significativamente con el uso de la mediación en las instituciones educativas públicas de la Red $\mathrm{N}^{\circ} 08$ UGEL N04 - Puente Piedra - Lima, 2017.

$H_{0}$. Los estilos de liderazgo del director no se relacionan significativamente con el uso de la mediación en las instituciones educativas públicas de la Red $\mathrm{N}^{\circ}$ 08UGEL Nº 04 -Puente Piedra - Lima, 2017.

Tabla 4. Correlación de la tercera hipótesis específica

\begin{tabular}{llcc}
\multicolumn{2}{c}{ Correlaciones } & Uso de la Mediación \\
\cline { 2 - 3 } \multirow{2}{*}{ Rho de Spearman } & Estilos de & Coeficiente de correlación & 0,474 \\
& liderazgo & Sig. (bilateral) & 0,000 \\
& & $N$ & 100 \\
\hline
\end{tabular}

Fuente: Zegarra (2017) 
Al aplicar la fórmula de Rho de Spearman, margen de error al $5 \%$, se halló un coeficiente de correlación moderada (Rho $=0,474$; $p<0,01)$. Por lo cual se rechaza la hipótesis nula y se acepta la hipótesis alterna, cuyos resultados fueron determinados por la respuesta de la muestra, en donde la mayoría considera que los estilos de liderazgo inciden en el uso de la mediación identificado como a veces adecuado.

\section{DISCUSIÓN}

La presente investigación científica determinó la relación entre los estilos de liderazgo del director y la gestión de conflictos en las instituciones educativas públicas de la Red $N^{\circ}$ 08- UGEL Nº4 - Puente Piedra - Lima, 2017. En la hipótesis general se halló una correlación muy buena entre las variables liderazgo del director y la gestión de conflictos en la población estudiada. Se halló diferencias en los resultados de la muestra, representadas en la variable liderazgo del director cuyas respuestas establecieron que el $86,0 \%$ considera que a veces cumple con sus expectativas, lejos del 9,0\% como casi nunca. Asimismo, para la variable gestión de conflictos las respuestas también se encuentran divididas, el 60,0\% lo refiere como a veces adecuado, seguido de un $31,0 \%$ adecuado. Con ello se manifiesta que es necesario establecer mejoras en los estilos de liderazgo directivo para reforzar la gestión de conflictos entre los integrantes de la institución.

Mediante el mismo análisis estadístico, en la primera hipótesis específica se determinó una correlación moderada entre variable estilos de liderazgo directivo y la dimensión manejo de la negociación de la población en estudio. Se encontró diferencias en la muestra, con respecto a la variable liderazgo del director cuyas respuestas establecieron que el $86.0 \%$ considera que a veces cumple con sus expectativas, lejos del 9,0\% como casi nunca. Asimismo, para la dimensión manejo de la negociación las opiniones oscilaron mayoritariamente entre $60,0 \%$ y $29,0 \%$ calificado como a veces adecuado y adecuado, respectivamente. Con lo cual se confirma la necesidad de reforzar los estilos de liderazgo directivo para cooperar en el manejo de la negociación entre los integrantes de la institución.

En la segunda hipótesis específica, las estadísticas encontraron un coeficiente de correlación moderada entre la variable estilos de liderazgo directivo y la dimensión comunicación cordial. Con este análisis se logró conocer las respuestas de la muestra, con respecto a la variable liderazgo del director cuyas respuestas establecieron que el $86.0 \%$ considera que a veces cumple con sus expectativas, lejos del 9,0\% como casi nunca. En el caso de la dimensión comunicación cordial sus opiniones discreparon mayoritariamente entre el $51,0 \%$ y $38,0 \%$ como a veces adecuado y adecuado, respectivamente. Con lo cual se confirma que la variable y la dimensión analizada necesitan reforzar los estilos de directivo que garantice la comunicación cordial dentro en beneficio de los integrantes de la institución.

De otro lado, en la tercera hipótesis específica representada por los estilos de liderazgo directivo y el uso de la mediación, establecieron una correlación moderada. Estos resultados se debieron a la variable el $86,0 \%$ considera que a veces cumple con sus expectativas, lejos del 9,0\% como casi nunca. Asimismo, para la dimensión uso de la mediación las respuestas también se encuentran divididas entre el $51,0 \%$ que lo identifica como a veces adecuado, seguido de un $38,0 \%$ como adecuado. Con lo cual se evidencia que entre la variable y la dimensión se necesita mejorar la aplicación de los estilos de liderazgo directivo en busca de mejoras en el uso de la mediación entre los integrantes de la institución en estudio.

En cuanto a los estudios antecesores a la presente investigación, notamos que Lizárraga (2015) destaca al estilo de liderazgo transformacional como el más importante para la eficacia educativa ya que es importante motivar y tener tolerancia psicológica con los integrantes de la institución educativa, asimismo destaca el liderazgo transaccional como principales fuentes para recibir incentivos cuando se cumpla idóneamente la tarea encomendada. Estos resultados difieren 
a los nuestros, ya que hemos comprobado que en una institución educativa se puede manifestar diferentes estilos de liderazgo directivo, los cuales generan insatisfacción la mayoría de veces, considerando el diálogo y la negociación como una de las principales fuentes perjudicadas.

En referencia a la gestión de conflictos, encontramos a Gómez (2015) quien el director gestiona el conflicto entre los integrantes de la institución de estudio mediante su estado de ánimo, si este es bueno será asertivo en sus emisiones verbales, expresándose de forma clara, precisa y conciliador; sin embargo, en situaciones de estrés o presión laboral, incluso en la toma de decisiones, emplea un estilo más agresivo acompañados de gestos y rasgos similares, autoritarios e imperantes. Aunque no hemos analizado las emociones del director cuando resuelve un conflicto, en la investigación se demuestra que el actuar del director no genera satisfacción en la mayoría porque carece de empatía, considerando necesario una actuación más acorde con la de líder, que solo guiarse por emociones.

Vidal (2017) en su tesis posgrado titulada "Estilos de liderazgo en una directora desde la percepción de los docentes de una institución educativa pública de la provincia constitucional del Callao" se planteó el objetivo de identificar y describir las características predominantes de los estilos de liderazgo que perciben los docentes en la directora de la población en estudio. En las conclusiones se destaca que identificó que los estilos de liderazgo predominantes son los estilos de liderazgo transformacional, transaccional y autoritario. $Y$ de manera no predominante el estilo de liderazgo laisses faire. Además, detalla que, desde la percepción de los docentes, que la directora presenta a menudo las características predominantes de estimulación intelectual, influencia idealizada, motivación e inspiración y consideración individualizada las cuales son características del estilo de liderazgo transformacional. Estos resultados no coinciden con el estilo de liderazgo que evidencia la investigación.

Los resultados hallados confirman discrepancias entre los variables estilos de liderazgo directivo y la gestión de conflictos, ya que la mayoría considera como a veces idóneo el liderazgo aplicado por el director, implicando con ello la gestión de conflictos calificado como a veces adecuado. Evidenciando la necesidad de identificar idóneamente el liderazgo directivo que permita afrontar los conflictos dentro de la institución de estudio.

La actitud del director frente a los conflictos debe ser eficiente y madura y esto sucederá cuando abandone su posición defensiva y pase a la acción preventiva y proactiva. Un enfoque de gestión positiva de conflictos se caracteriza por el diálogo, la inclusión y el respeto hacia todas las personas, un alto nivel de confianza y libres de cualquier tipo de presión, imposición o coerción; de esta manera, la gestión de los conflictos que se presentan cotidianamente en las instituciones educativas serán eficientes y se trabajará en un ambiente donde todos se sientan seguros y valorados.

\section{AGRADECIMIENTO}

Al Dr. Abelardo Campana Concha por su asesoramiento y apoyo constante durante todo el proceso de investigación. 


\section{REFERENCIAS BIBLIOGRÁFICAS}

Alvarado, Otoniel. (2003). Gerencia y marketing educativo: Herramientas modernas de gestión educativa. Perú: Universal Alas Peruanas.

Gómez, Beatriz. (2015). La comunicación en la resolución de conflictos entre los docentes y la gerencia escolar. Tesis para optar el grado de Magister. Universidad de Carabobo, Venezuela.

Mena, Doris. (2017) El rol del director en la gestión de conflictos en el marco de buen desempeño del directivo. Tesis para optar el grado de Maestría. Universidad de Piura, Piura.

Minedu (2009). Aprendiendo a resolver conflictos en las instituciones educativas. Tutoría y orientación educativa. Perú: Quebecor Word Perú, SA.
Lizárraga, José. (2015). Estilo de liderazgo del director y la eficacia de la institución educativa "Pedro Ruiz Gallo"-2015. Tesis para optar el grado académico de Doctor. Universidad Nacional de Educación Enrique Guzmán y Valle, Lima, Perú.

Vidal, Manuel. (2017). Estilos de liderazgo en una directora desde la percepción de los docentes de una Institución Educativa Pública de la Provincia Constitucional del Callao. Tesis para optar el grado de Magister. Pontificia Universidad Católica del Perú, Lima, Perú.

\section{Correo electrónico:}

luzangelicaz@hotmail.com

Revisión de Pares:

Recibido: 20 - 04 - 2018

Aceptado: 25 - 06 - 2018 